\title{
A Novel Intrusion Detection System for Internet of Healthcare Things Based on Deep Subclasses Dispersion Information
}

This paper was downloaded from TechRxiv (https://www.techrxiv.org).

LICENSE

CC BY-NC-SA 4.0

SUBMISSION DATE / POSTED DATE

02-03-2022 / 03-03-2022

\section{CITATION}

Fouda, Marwa; Ksantini, Riadh; Elmedany, Wael (2022): A Novel Intrusion Detection System for Internet of Healthcare Things Based on Deep Subclasses Dispersion Information. TechRxiv. Preprint. https://doi.org/10.36227/techrxiv.19292444.v1

$\mathrm{DOI}$

10.36227/techrxiv.19292444.v1 


\title{
A Novel Intrusion Detection System for Internet of Healthcare Things Based on Deep Subclasses Dispersion Information
}

\author{
Marwa Fouda (D), Riadh Ksantini (ID, and Wael Elmedany (D)
}

\begin{abstract}
Despite the significant benefits that the Internet of Healthcare Things (IoHT) offered to the medical sector, there are concerns and risks regarding these systems which can delay their wide deployment as they handle sensitive, and often life-critical medical information. In addition to the IoHT concerns and risks, there are security constraints which include hardware, software, and network constraints that pose a security challenge to these systems. Therefore, security measures need to be deployed that can overcome the concerns, mitigate the risks, and meet the constraints of the IoHT. For these reasons, a subclasses intrusion detection system for the IoHT is proposed in this research work based on a novel variation of the standard One-class Support Vector Machine (OSVM), namely Deep Subclass Dispersion Oneclass Support Vector Machine (Deep SDOSVM), which considers subclasses in the target class, i.e. normal class, in order to minimize the data dispersion within and between subclasses, thereby improving the discriminative power and classification performance of the intrusion detection system. A deep clustering model is used for subclasses generation in the proposed Deep SDOSVM approach, namely the Dynamic Autoencoder Model (DynAE), to overcome the drawbacks of the classical clustering algorithms, and further enhance the classification performance of the intrusion detection system. The proposed deep clustering subclasses intrusion detection system was evaluated on the realworld TON_IoT dataset, and compared to other state-of-theart one-class classifiers. Experimentation results showed that the proposed approach outperformed the other relevant one-class classifiers for network intrusion detection.
\end{abstract}

Index Terms-Data Dispersion, Deep Clustering, Internet of Healthcare Things, Internet of Things, Intrusion Detection, Oneclass Classification, Subclasses Information

\section{INTRODUCTION}

$\mathbf{R}$ ECENT progression in computer technologies, and the fast expansion of the Internet lead to the evolution of the Internet of Things (IoT) [1], [2]. The IoT allows the smooth integration of both physical and digital worlds through a network of physical objects embedded with sensors [3]. Such a paradigm gave rise to smart environments like smart cities, smart homes, smart transportation, etc. [2]

The Internet of Healthcare Things (IoHT), a subdomain of the IoT, is an ecosystem of various connected devices which

(Corresponding author: Marwa Fouda.)

Marwa Fouda is with the Department of Information Systems, College of Information Technology, University of Bahrain, Sakhir, Southern Governorate, Kingdom of Bahrain (e-mail: marwa.fouda.2021@gmail.com).

Riadh Ksantini is with the Department of Computer Science, College of Information Technology, University of Bahrain, Sakhir, Southern Governorate, Kingdom of Bahrain.

Wael Elmedany is with the Department of Computer Engineering, College of Information Technology, University of Bahrain, Sakhir, Southern Governorate, Kingdom of Bahrain. includes sensors, wearable devices, medical devices, and clinical systems that enable several applications in the medical sector like remote patient monitoring, fitness programs, and chronic diseases and elderly care. The IoHT devices collect the patient's real-time and sensitive data, transfer the collected data through a gateway for storage in a cloud platform, and redirect the stored data to the required healthcare providers [2]-[4].

The IoHT has benefited the medical sector through remote monitoring of the patient's health status without any visits by him/her to the healthcare facility. This resulted in time and cost savings, enhanced quality of healthcare services, improved quality of patient's health through better support and medication as healthcare providers can effectively and accurately understand critical health conditions, patients becoming well-informed about the improvements in their health status and treatments, and increased patient interest and satisfaction through simple and effective communication with healthcare providers [1]-[4].

Despite the aforementioned benefits of the IoHT, there are security and privacy concerns regarding this subdomain which can delay its wide deployment as such systems handle sensitive, and often life-critical medical information. Cyberattacks on the IoHT and its components can threaten its confidentiality, integrity, availability, and authentication which can result in severe physical and life-threatening harm to the patients. For example, hacking a medical insulin pump can result in insulin over dose, and possible death of the patient. Also, hacking a connected cardiac device, for example a pacemaker, can threaten a patient's life [2], [3].

The security of the IoHT is also accompanied with constraints that need to be considered, and are categorized to hardware, software, and network constraints. The hardware constraints include computational, energy, and memory constraints. The software constraints include embedded software constraints, and dynamic security patch. Finally, the network constraints include scalability, diverse IoT devices, diverse communication medium, multi-protocol networking, and dynamic network topology [5].

Machine Learning (ML) intrusion detection systems became the most promising technique for securing the IoHT. This technique can recognize attacks especially unknown or zeroday attacks that traditional intrusion detection systems are unable to identify through the detection of variations in the characteristics of network traffic. Even though ML may not be a convenient choice for problems requiring a formal 
descriptive solution, strong results can be achieved by it in areas and issues that can be difficult to formalize. Therefore, ML is proficient in both data clustering and classification fields which are the main blocks in data security applications [6], [7].

Most Internet security models block malicious behaviors using a list of these behaviors. However, attackers continuously improve their techniques making it difficult to detect their adaptive malicious behaviors by these models. Therefore, describing all possible malicious behaviors in a list, and continuously updating that list in these models is considered impractical, and extremely resource-intensive. ML can model normal behavior such that any incoming event that deviates from the modeled normal behavior is considered an anomaly which is more likely a malicious behavior. This makes ML a more practical security approach. Also, ML intrusion detection systems have low to medium resource consumption, and therefore, resources are used effectively [6], [7].

The growing demands and growth of the IoHT require improvements in security solutions. Therefore, a ML analytical tool is often used to analyze the massive bulk of data, and provide worthy knowledge in the classification, decision analysis, and cyber-attacks detection [2].

ML algorithms use training and learning from experience to improve their performance in fulfilling a task. For a ML intrusion detection system, the task is to classify a system behavior as normal or abnormal. To improve the performance of this system, classification accuracy needs to be improved, and the collection of normal behaviors are the experiences that the ML algorithms learn from [8].

Traditional network intrusion detection systems experience high false detection rates that can be reduced using ML techniques. A ML intrusion detection system uses statistical, genetic, and heuristics or a combination of them to improve the detection rate, and reduce false negatives through dissemination of complex attack patterns [6].

ML one-class intrusion detection systems have drawn attention in the IoHT to overcome the drawbacks of both binary and multi-class ML intrusion detection systems which include the high cost of data labelling, and the class imbalance problem. However, ML one-class intrusion detection systems for the IoHT did not consider subclasses data distribution for the normal behavior in the IoHT environment, where the definition of normal behavior can vary from one system to another in these environments. Also, the ML binary class and multiclass hierarchy subclasses intrusion detection systems did not consider the subclasses dispersion information.

Classical clustering algorithms, such as K-means, have been used to generate the subclasses based on the optimal number of clusters for the target class. These clustering algorithms grasp similarities between data points using distance-based metrics, and therefore, are considered shallow models. Such models fail to achieve reliable discriminative power, i.e. using distance-based metrics is not adequate for the discovery of representative similarities between data points. Moreover, the quadratic complexity of some of the shallow models is considered a restriction for processing large-scale datasets similar to those generated by the IoHT environments. And since data clustering is done in raw data space, the shallow models cannot cope with the curse of dimensionality [9].

Therefore, a novel ML subclasses intrusion detection system for the IoHT is proposed that presents a novel variation of the standard One-class Support Vector Machine (OSVM) for classification, namely, the Deep Subclass Dispersion One-class Support Vector Machine (Deep SDOSVM). The proposed Deep SDOSVM approach considers the subclasses in the IoHT normal/target data class, in order to minimize the data dispersion within and between subclasses thus improving the discriminative power and classification performance of the intrusion detection system.

Deep clustering is used for subclasses generation in the proposed Deep SDOSVM approach for several reasons. First, large-scale datasets like the IoHT datasets can be handled using the multi-layer architecture of these models because of the mini-batch Stochastic Gradient Descent (SGD) that gives the neural networks the capacity to evade both time and memory bottlenecks. Second, the ability of neural networks to project data from high-dimensional space to low-dimensional space. Finally, the neural networks can discover representative similarities between data points, and therefore, achieve a better discriminative power than shallow models [9].

Most of the existing deep clustering methods use an autoencoder architecture, where a reconstruction cost regularizes the encoder training, and the decoder is utilized to mitigate the training effect with pseudo-labels. However, the elimination of the decoding network, and the usage of hypothetical similarities to train the encoder can cause easily the corruption of the space topology. This can possibly cause the encoder to generate random discriminative features that do not represent the real discriminative characteristics. Such a problem is referred to as Feature Randomness [9].

Providing autoencoders with their reconstruction capability in order to capture notable features without imposing any randomness can alleviate the Feature Randomness problem. However, combining both clustering and reconstruction can be a problem because of the natural trade-off between them. The aim of the clustering objective function is the destruction of non-discriminative details, whereas the aim of the reconstruction objective function is preserving all information, i.e. all information will have the same importance regardless whether they are relevant or not for clustering. Such a problem is referred to as Feature Drift [9].

To address both the Feature Randomness and Feature Drift problems, a novel deep clustering model proposed by [9], namely the Dynamic Autoencoder (DynAE), is used to handle a clustering-reconstruction trade-off through gradual and smooth elimination of the reconstruction step, using a dynamic loss function.

To the best of knowledge, this research work is the first to explore the usage of deep clustering subclasses for one-class classification in anomaly-based IoHT network intrusion detection in order to incorporate IoHT data subclasses information related to diverse normal behaviors, and improve detection accuracy.

The paper is organised as follows: Section $[\mathrm{II}$ discusses the related work to the IoT and IoHT ML intrusion detection systems. Section III provides a detailed overview of the 
proposed Deep SDOSVM intrusion detection system. Section IV presents the experimental datasets and protocol as well as the experimentation results, and their discussion. Section $\mathrm{V}$ concludes the paper with future remarks.

\section{RELATED WORK}

In this section, we provide a review of state-of-the-art ML intrusion detection systems for IoT and IoHT. A summary of the reviewed ML intrusion detection systems is illustrated in Table [. We also summarize subclass intrusion detection solutions, and provide our contribution.

The thematic taxonomy of the ML intrusion detection systems for the IoHT shown in Fig. 1 is discussed in the following subsections. The blue boxes in the figure demonstrate the classification of the proposed approach in each level.

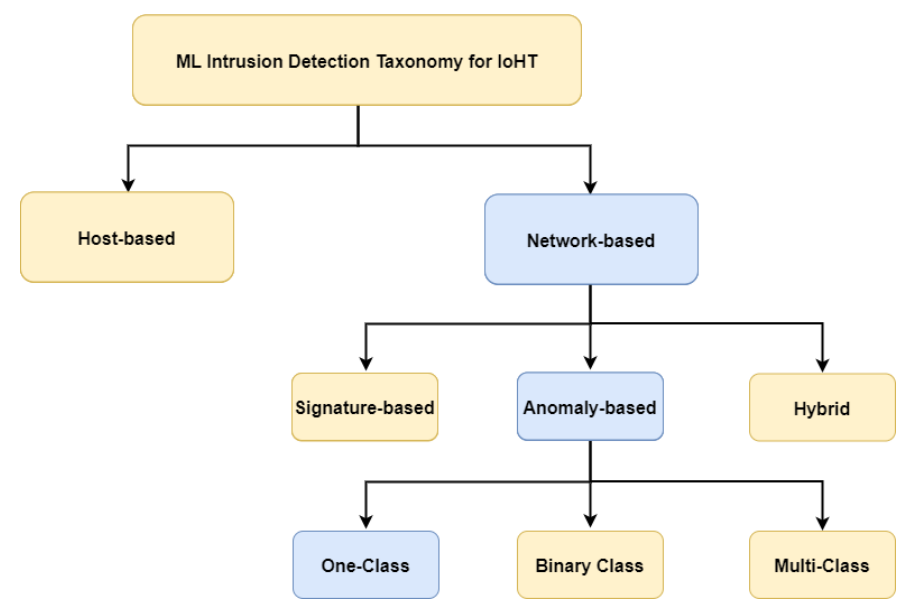

Fig. 1. ML intrusion detection taxonomy for the IoHT

\section{A. Host-based and Network-based Intrusion Detection Sys- tems}

The IoT and IoHT intrusion detection systems can be categorized based on the data source to Host-based Intrusion Detection System (HIDS) and Network-based Intrusion Detection System (NIDS).

According to [6], the HIDS is suitable for inside intrusion detection. Also, [14] used the HIDS approach to overcome the drawback of the NIDS, where the end-to-end encryption is used in most network communications, thus making the acquisition of useful information from the network traffic more difficult.

In [14], a host-based Internet of Things Detection System (IoTDS) was proposed which depends on the host device resource consumption data due to its sensitivity to the presence of bot malware, and therefore, it was able to detect the malware present within the host device accurately. Also, because of its dependency on the host device resource consumption data, the IoTDS malware detection capability was not obstructed by the encryption of malware traffic. The authors considered the impact of IoTDS on the host device's performance where the experiments showed that the host device remained functional despite the increase in resource consumption when the intrusion detection system was run on the host device. The authors stated that pre-installation of IoTDS can be done in IoT devices, thus providing out of the box protection to these devices. The authors also recommended the combination of other types of data, such as network-based data, with hostbased data for a more secure system that includes both the hosts and the network.

However, [6] did not recommend the HIDS for the heterogeneous and large IoT systems due to the per-device security approach of the HIDS, where each device is analyzed separately. Also, [23] did not recommend the use of the HIDS for several reasons. First, the IoT manufacturers cannot be trusted to install the required HIDSs on the IoT devices. Second, the limited access to some of the IoT devices, such as wearables, is another drawback of the HIDS as it is not possible to compel their installation on these devices. Finally, the HIDS consumes resources when deployed in the resource-constraint IoT devices, and harms their functionality. Therefore, these systems need to be lightweight with respect to the resourceconstraint IoT devices, and preserve their functionality.

On the other hand, the NIDS is suitable for external intrusion detection. Also, these systems offer more degree of freedom than the HIDS for developing security solutions as they are suitable for the resource-constraint IoT systems. The NIDS can be used as a single system or multiple systems that can monitor the entire IoT network, making them suitable for large and heterogenous IoT systems [6].

The IoHT is a growing and heterogeneous environment, and consists of resource-constraint devices. Therefore, for our proposed intrusion detection system we recommend a NIDS to meet the IoHT security constraints mentioned in section I

\section{B. Intrusion Detection Methods}

The intrusion detection methods for the IoT and IoHT can be categorized to signature-based, anomaly-based, and hybrid.

In the signature-based detection, also known as abuse detection or rule-based detection or misuse-detection, predefined attack patterns are modeled, and preserved in the attacks' signature database. If the pattern of an incoming traffic matches any of the attacks' signatures in the database, then the administrator is alerted [2], [22].

Signature-based detection can detect known attacks, and thus has high detection rates for these attacks. However, it suffers from poor performance in detecting unknown attacks. Also, another drawback of signature-based detection is that it is not suitable for real-time intrusion detection because any increase in the number of newly recognized attacks results in an increase in the number of signatures, and therefore, more comparisons will be required between the stored signatures and incoming events. This can cause an overload for the intrusion detection system, and thus affects its response. Therefore, these systems neglect the examination of incoming events at specific rates based on the available processing resources. Furthermore, signature-based detection requires human experts to develop signatures for new attacks, and this can consume time in order to study the characteristics of the new attack and generate its signature [17], [23]. 
TABLE I

SUMMARY OF STATE-OF-THE-ART ML INTRUSION DETECTION SYSTEMS FOR IOT AND IOHT

\begin{tabular}{|c|c|c|c|c|}
\hline Reference & Data Source & Classifier & Intrusion Detection Method & Algorithm Used \\
\hline [10] & Network & Binary class & Anomaly-based & $\begin{array}{l}\text { Deep AutoEncoder-Deep Feed Forward Neural Network } \\
\text { (DAE-DFFNN) }\end{array}$ \\
\hline$[11]$ & Network & Binary class & - & $\begin{array}{l}\text { Ensemble of Online Sequential-Extreme Learning Machine } \\
\text { (EOS-ELM) }\end{array}$ \\
\hline$|12|$ & Network & 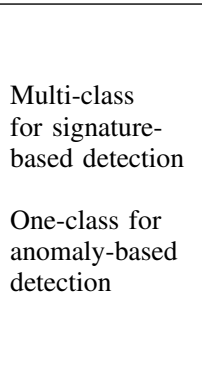 & Hybrid & $\begin{array}{l}\text { For signature-based detection, three approaches were used: } \\
\text { Approach (1): Supervised algorithms (J48, Bayesian Network } \\
\text { (Bayes Net), Random Forest (RF), and Hoeffding tree) } \\
\text { Approach (2): Multi-layer Neural Network (NN) using } \\
\text { backpropagation learning } \\
\text { Approach (3): Distributed classification using Adaptive } \\
\text { Boosting (AdaBoost) algorithm } \\
\text { For anomaly-based detection: OSVM }\end{array}$ \\
\hline$[13]$ & Network & Multi-class & - & J48 Decision Tree (DT) \\
\hline $\mid 14$ & Host & One-class & Anomaly-based & $\begin{array}{l}\text { Elliptic Envelope (EE), Isolation Forest (IF), } \\
\text { Local Outlier Factor (LOF), OSVM }\end{array}$ \\
\hline $\mid 15$ & Network & $\begin{array}{l}\text { Multi-class } \\
\text { One-class }\end{array}$ & Anomaly-based & $\begin{array}{l}\text { Autoencoder, IF, OSVM, RF, Single-Objective Generative } \\
\text { Adversarial Active Learning (SOGAAL), K-means clustering }\end{array}$ \\
\hline$|16|$ & $\begin{array}{l}\text { Operational data } \\
\text { in smart grids }\end{array}$ & One-class & Anomaly-based & $\begin{array}{l}\text { OSVM, IF, Angle-Base Outlier Detection (ABOD), } \\
\text { Stochastic Outlier Selection (SOS), } \\
\text { Principal Component Analysis (PCA), } \\
\text { deep fully connected autoencoders }\end{array}$ \\
\hline$[17]$ & Network & One-class & Anomaly-based & IF, LOF \\
\hline[ & Network & Binary class & - & - \\
\hline$|7|$ & $\begin{array}{l}\text { Network and } \\
\text { patient's } \\
\text { biometric }\end{array}$ & Binary class & Anomaly-based & $\begin{array}{l}\text { Artificial Neural Network (ANN), } \\
\text { K-Nearest Neighbor (KNN), Linear SVM, RF }\end{array}$ \\
\hline [18] & Network & Multi-class & Pre-defined threshold & Q-learning \\
\hline$|19|$ & Network & One-Class & Hybrid & $\begin{array}{l}\text { For signature-based detection: C5.0 DT } \\
\text { For anomaly-based detection: OSVM }\end{array}$ \\
\hline |2| & Network & Binary class & Anomaly-based & $\begin{array}{l}\text { Ensemble learning with two learning levels: } \\
\text { First level uses three learning algorithms: } \\
\text { DT, Naive Bayes (NB), RF } \\
\text { Second level uses XGBoost }\end{array}$ \\
\hline$|20|$ & Network & Binary class & - & $\begin{array}{l}\text { Hybrid Deep Learning (DL): cuda Deep Neural Network } \\
\text { Long Short Term Memory-Convolutional Neural Network } \\
\text { (cuDNNLSTM-CNN) }\end{array}$ \\
\hline$[\overline{4}]$ & Network & Binary class & Anomaly-based & Deep Neural Network (DNN) \\
\hline [21] & Network & One-class & Anomaly-based & OSVM \\
\hline $\mid 22$ & Network & Multi-class & Hybrid & $\begin{array}{l}\text { For signature-based detection: Modified Optimum Path } \\
\text { Forest (MOPF) } \\
\text { For anomaly-based detection: Optimum Path Forest } \\
\text { Clustering (OPFC) }\end{array}$ \\
\hline 23 & Network & One-class & Anomaly-based & OSVM \\
\hline 3 & $\begin{array}{l}\text { Network and } \\
\text { device }\end{array}$ & $\begin{array}{l}\text { Multi-class } \\
\text { and binary class } \\
\text { for network } \\
\text { level detection } \\
\text { One-class for } \\
\text { device level } \\
\text { detection }\end{array}$ & Anomaly-based & $\begin{array}{l}\text { DT, KNN, NB, RF, SVM for network level detection } \\
\text { Polynomial Regression for device level detection }\end{array}$ \\
\hline $\mid 24$ & Network & $\begin{array}{l}\text { Binary class } \\
\text { for gateway } \\
\text { level detection } \\
\text { One-class for } \\
\text { Wireless Sensor } \\
\text { Network } \\
\text { (WSN) level } \\
\text { detection }\end{array}$ & Anomaly-based & $\begin{array}{l}\text { For WSN detection level: OSVM } \\
\text { For gateway detection level: Deep learning }\end{array}$ \\
\hline
\end{tabular}


Because of the aforementioned drawbacks of the signaturebased detection method, anomaly-based and hybrid detection methods emerged as an alternative for ML intrusion detection systems in the IoT and IoHT.

In the anomaly-based detection, a typical normal network behavior is modeled, and any deviations between incoming network traffic and normal network behaviors or system activities is considered an anomaly which is most likely an intrusion [2], [22].

Anomaly-based detection can detect both known and unknown attacks because it assumes that the difference between normal and abnormal behavior can be detected [23].

The anomaly-based detection was used by [2] because IoHT environments are heterogeneous and diverse, which practically limits the usage of a signature-based detection method in the protection of these environments from cyber-attacks. Therefore, this method is preferable for designing a strong intrusion detection system.

In [4], the anomaly-based detection was preferred than the signature-based detection for their intrusion detection system, because it provides a scalable solution to handle the dynamic and random behaviors of malicious attacks in an IoHT environment, thus making their system effective and efficient in classifying and detecting unexpected attacks. A lightweight anomaly-based approach was used by [17] in their Passban intrusion detection system for the same reason because of its capability of detecting new attacks without any hardware upgrades making it an effective approach for resource-constraint IoT devices.

However, a major challenge for anomaly-based detection is the difficulty of generating a model for a singular normal behavior of a system which consists of diverse behaviors created by various data sources. This results in high False Positive Rate (FPR) because of the minimal likeness between incoming events and modeled normal behaviour [17].

In [19], both signature-based and anomaly-based detection methods were used for their two stage novel hybrid intrusion detection system that takes advantage of both methods, and overcomes their limitations. In the first stage of the hybrid intrusion detection, the signature-based detection was used to identify known attacks. In the second stage, the anomaly-based detection was used to identify unknown or zero-day attacks without having a negative impact on the energy consumption of the IoT node due to the low processing costs of this method. This lead to improvement in the True Positive Rate (TPR), and reduction of the FPR compared to the TPR and FPR performance for each approach implemented individually. Also, for the Signature Intrusion Detection System (SIDS) to have an updated history of known attacks, the attacks recognized by the Anomaly Intrusion Detection System (AIDS) were saved in the SIDS database for protection from similar attacks in the future.

For our proposed intrusion detection system, since the one-class classifier models normal network behaviors, and identifies any event that deviates from that normal behavior as an outlier which is most likely an intrusion, the anomalybased detection is used for the classification problem. Also, to overcome the challenge of this detection method mentioned previously, a subclasses approach is proposed in order to incorporate the IoHT subclasses information related to diverse normal behaviors, and improve the FPR.

\section{Classifiers}

The classifiers used in the IoT and IoHT can be categorized to one-class, binary class, and multi-class classifiers.

One-class classifiers, a special type of ML technique, model a single pattern, i.e. all data samples in the training dataset belong to a single class of interest usually the normal class for the intrusion detection system, and then that model is used to recognize if a new data sample belongs to that pattern or class. On the other hand, both binary class and multi-class classifiers recognize a new data sample as belonging to one of two or more pre-defined patterns, respectively [14], [17].

Binary class and multi-class classifiers for the IoT and IoHT intrusion detection systems require labelled data, where each label represents a class of interest, when training a model that has the ability of detecting each class [14].

The above requirement is considered a drawback for these classifiers for the following reasons [14], [19]:

1) Cost of data labelling can be too high: For the case of malware detection, testbeds or honeypots that are designed specially for this purpose are used to collect the labelled data which requires vulnerable devices, and a special kind of access to malware to be done manually.

2) Class imbalance problem: Normal class data is available, and in larger scale unlike the malicious class data which are rare or do not even exist.

Because of the aforementioned drawbacks of both binary class and multi-class classifiers, one-class classifiers emerged as an alternative for the IoT and IoHT intrusion detection systems where a new model is trained using the normal data only as the target class, that is easily collected than the malicious data which is unavailable or poorly sampled or not well defined, and that model recognizes a new instance as normal if it fits within the boundaries of the model or malicious if it doesn't [14], [19].

As a result [14], [17]:

1) Labelling costs as well as the necessity for malicious data samples in the training phase will be reduced significantly.

2) Easy adapting models, as periodical updates of the model using new normal data requires less effort than updates that use labelled normal and malicious data.

3) Effective models for resource-constraint IoT devices.

4) Suitable models for instant detection of anomalies in real-time network traffic monitoring due to the low computational cost of the model inference.

In [14], the one-class classifier was used for their IoTDS to address the issues of both the binary class and multi-class classifiers which is the dependency on algorithms that need labelled data to train the model, and this can be a problem as it is difficult to obtain labelled data for botnets attacks as well as this data can become quickly outdated by new botnets and attacks. 
In [17], the lightweight Passban one-class anomaly-based intrusion detection system provides protection near the IoT network edge. One-class classification algorithms were used as they proved to be efficient for resource-constraint IoT devices.

The authors in [21] used the OSVM for their detection model for several reasons. First, its low energy consumption for the WSN when compared to other ML algorithms. Second, it was considered a convenient method to detect selective forward and jamming attacks. Finally, it works well with structured data as a table of values when compared to other ML algorithms.

Hierarchical Anomaly Detection and Localization (HADL) was proposed by [24] in which a lightweight OSVM was used for detecting WSN routing attacks. It is executed from parent to child nodes on-demand in the WSN, and hence continuous network monitors are not required which reduces energy consumption and processing time.

However, these one-class intrusion detection systems for the IoT and IoHT did not consider subclasses data distribution for the normal behaviors of these environments where the definition of normal behavior can vary from one system to another in these environments.

\section{Subclasses Intrusion Detection Solutions}

In [25], an autonomous two stage intrusion detection system based on hierarchical subclasses data distribution named Soft Computing-based Anomaly Detection (SCAD) was proposed. The authors proved through experimentation the effectiveness of their system for both binary and multi-class classification. However, to reduce the training time the authors recommended the use of GPU and parallel processing. Also, the authors did not consider the subclasses dispersion information in order to improve the classification performance of their intrusion detection system.

Therefore, we propose a novel ML subclasses one-class anomaly-based network intrusion detection system for the IoHT that presents a novel variation of the standard OSVM for classification, namely the Deep SDOSVM. The proposed Deep SDOSVM approach considers the subclasses in the IoHT normal data class, which is considered the target class in this paper, in order to minimize the data dispersion within and between subclasses thus improving the discriminative power and classification performance of the intrusion detection system. Deep clustering is used for subclasses generation in the proposed Deep SDOSVM approach to overcome the drawbacks of the classical clustering algorithms mentioned previously in Section II, and further enhance the classification performance of the intrusion detection system.

\section{Proposed DeEp SDOSVM InTRusion Detection SYSTEM}

In this section, we describe the proposed Deep SDOSVM intrusion detection system in details. The proposed system consists of three steps: feature pre-processing, deep clustering using the DynAE model, and classification using the proposed SDOSVM algorithm as illustrated in Fig. 2 .

\section{A. Feature Pre-processing}

This subsection presents in detail the steps involved in feature pre-processing. The IoHT network traffic generated by various sensors has different types of features that include numerical and categorical values, and pre-processing these features as described below is important so that the proposed intrusion detection system can achieve accurate and efficient results [2].

1) Feature Mapping: The IoHT network traffic consists of numerical and categorical features. The values of categorical features need to be transformed to numeric ones because the proposed intrusion detection system can handle efficiently only numeric values [2].

2) Feature Selection: This method involves the identification of proper features, and dismissing the irrelevant ones in order to obtain a features subset that best represent the classification problem, and with minimum performance degradation in the IoHT environment. The advantages of such a method include improvement in the performance of the intrusion detection system, and reduction in processing time [2].

3) Feature Normalisation: The features of the IoHT traffic have dissimilar ranges of values. Therefore, these features need to be normalized, i.e. convert every value in a particular feature to a value in the data range $[0,1]$. The advantages of this method include speeding up the gathering and removal of bias from the raw data, ease pattern analysis, and support convergence and training time [2], [3], [11].

The following equation was used to normalize the features of the IoHT data:

$$
F_{\text {Normalized }}=a+\frac{F-F_{\min }}{F_{\max }-F_{\min }}(b-a),
$$

where

$F_{\text {Normalized }}$ are the new values for a feature $F$ in $X$ after normalization,

$a$ is the minimum value for the normalized data range, i.e. 0 , $b$ is the maximum value for the normalized data range, i.e. 1, $F$ is the feature to be normalized in $X$, $F_{\text {min }}$ is the minimum value for feature $F$, $F_{\max }$ is the maximum value for feature $F$, and $X=\left\{x_{i}\right\}_{i=1}^{N}$ is the training dataset of $\mathrm{N}$ samples.

\section{B. Deep Clustering using DynAE}

This subsection presents in detail the steps involved in the generation of the subclasses for the target class using the DynAE.

The DynAE is a novel deep clustering model proposed by [9] that handles a clustering-reconstruction trade-off through gradual and smooth elimination of the reconstruction objective function in favor of a construction one using a dynamic loss function. The DynAE architecture is illustrated in Fig. 3

The DynAE deep clustering approach consists of two steps: pretraining and clustering.

1) Pretraining: The autoencoder is initially pretrained, i.e. both the encoder and decoder are trained, and the learning weights are generated. 


\section{Deep SDOSVM Intrusion Detection System}

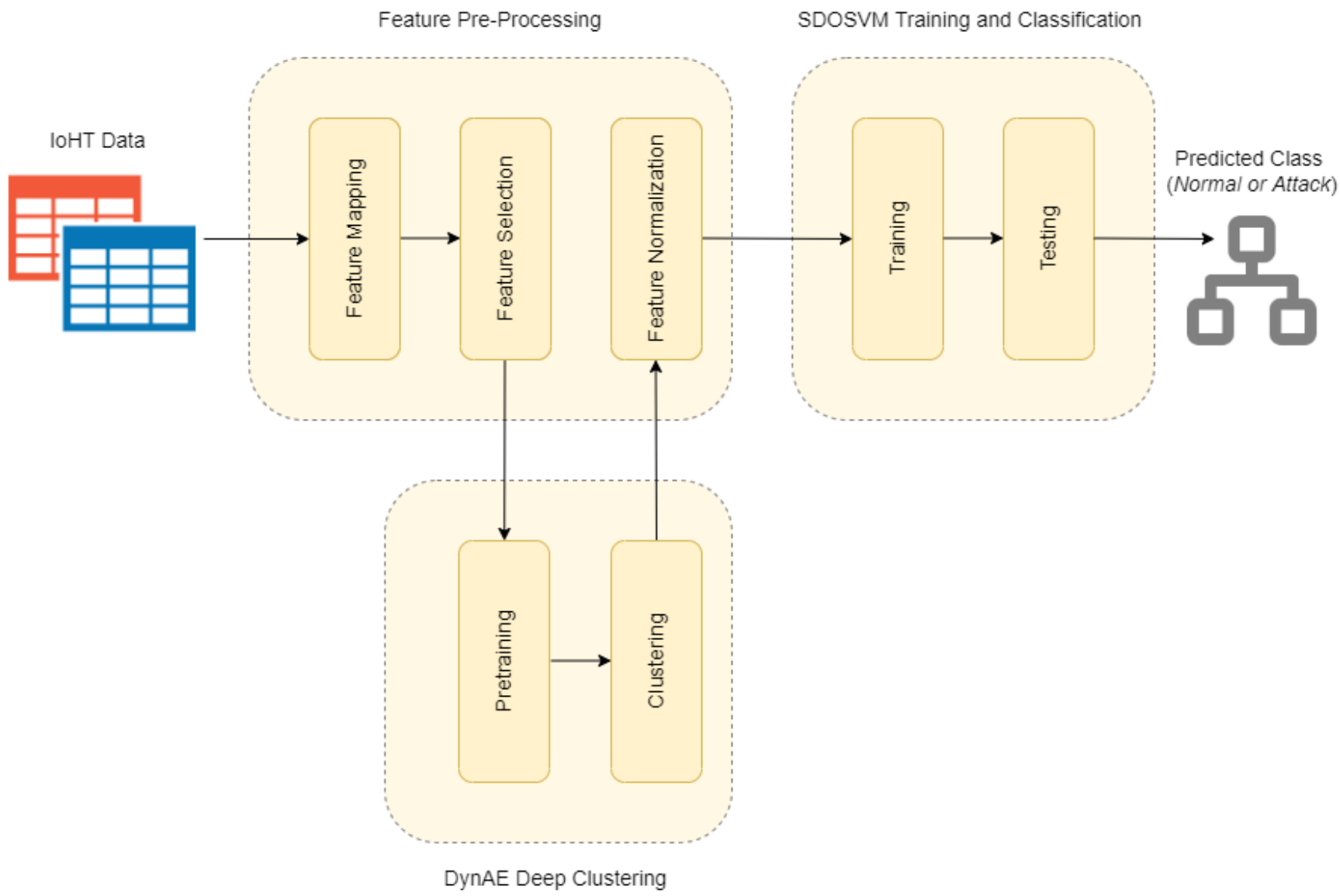

Fig. 2. The proposed Deep SDOSVM intrusion detection system

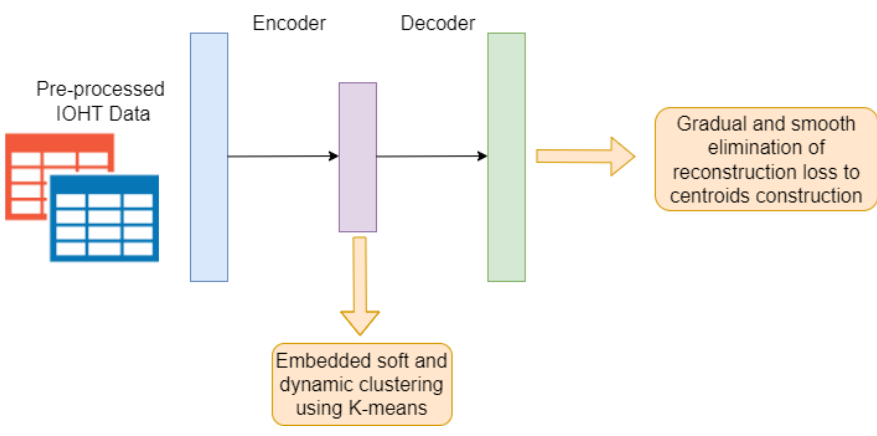

Fig. 3. The DynAE architecture

2) Clustering: After pretraining, the autoencoder weights are finetuned through the optimization of a dynamic loss function.

At the beginning, the embedded clusters' centers are initialized using K-means, and a clustering assignment matrix $C=c_{i j}$ is calculated as follows:

$$
c_{i j}=\frac{\left(1+\frac{\left\|z_{i}-e_{j}\right\|^{2}}{A}\right)^{-\frac{A+1}{2}}}{\sum_{j^{\prime}}\left(1+\frac{\left\|z_{i}-e_{j^{\prime}}\right\|^{2}}{A}\right)^{-\frac{A+1}{2}}},
$$

where $c_{i j}$ represents the probability of assigning an embedded data point $z_{i}$ to an embedded centroid $e_{j}$, and $A$ represents the Student's t-distribution coefficient.

After the pretraining step, the clustering assignments $c_{i j}$ for some of the embedded data points $z_{i}$ are unreliable, i.e. the probabilities of their highest clustering assignments are very close to each other. These data points are very close to the cluster boundaries, and predicting their corresponding centers confidently is very difficult. Therefore, these data points are considered conflicted data points.

An unconflicted data point is characterized as belonging to the set $S_{\text {unconf }}$ by the following condition:

$$
S_{\text {unconf }}=\left\{x_{i} \in X \mid \eta_{i 1}>B_{1} \text { and }\left(\eta_{i 1}-\eta_{i 2}\right)>B_{2}\right\},
$$

where

$\eta_{i 1}$ represents the highest cluster assignment probability for a data point,

$\eta_{i 2}$ represents the second highest cluster assignment probability for a data point,

$B_{1}$ represents the minimum confidence threshold under which a data point is considered conflicted, and $B_{2}$ represents the minimum difference between the highest and second highest cluster assignment probabilities. 
$B_{1}$ and $B_{2}$ are trade-off hyperparameters that belong to the interval $[0,1]$, and are calculated as follows:

$$
B_{1}=\frac{\kappa}{N_{\text {sub }}} \text { and } B_{2}=\frac{B_{1}}{2}, \kappa \in\left[\left|1, N_{\text {sub }}\right|\right],
$$

where

$\kappa$ represents the confidence threshold,

and $N_{s u b}$ is the number of subclasses or clusters.

During each training iteration of the DynAE, the number of conflicted data points should decrease gradually. The dynamic loss function stabilizes when there is no more decrease in the number of conflicted data points.

\section{Classification using SDOSVM Algorithm}

This subsection presents in detail the mathematical formulation of the proposed SDOSVM algorithm, using linear and nonlinear kernel functions, in order to handle linear and nonlinear decision boundaries between data.

1) Linear SDOSVM: The proposed SDOSVM algorithm is a novel variation of the standard OSVM that considers subclasses in the target class in order to minimize the data dispersion within and between subclasses thus improving the discriminative power and classification performance. To achieve this, the within and between subclasses matrix of (5) will be added to the standard OSVM primal optimization problem of (8).

The within and between subclasses matrix $M_{s u b}$ can be formulated as:

$$
M_{s u b}=M_{W}^{s u b}+M_{B}^{s u b}
$$

where

$M_{W}^{s u b}$ represents the within subclass matrix,

and $M_{B}^{s u b}$ represents the between subclasses matrix.

$$
\begin{aligned}
& M_{W}^{s u b} \text { and } M_{B}^{s u b} \text { can be formulated as: } \\
& \qquad M_{W}^{s u b}=\sum_{i=1}^{N_{s u b}} \sum_{j=1}^{N_{i}} p_{i}\left(x_{j}-\mu_{i}\right)\left(x_{j}-\mu_{i}\right)^{T},
\end{aligned}
$$

where

$N_{i}$ is the number of samples that belong to the $\mathrm{i}^{\text {th }}$ subclass, $p_{i}=\frac{N_{i}}{N}$ is the prior probability of the $\mathrm{i}^{\mathrm{th}}$ subclass, $\mu_{i}$ is the mean vector of the $\mathrm{i}^{\text {th }}$ subclass, and $x_{j}$ is the training sample of the $\mathrm{i}^{\text {th }}$ subclass.

$$
M_{B}^{s u b}=\sum_{i=1}^{N_{s u b}}\left(\mu_{i}-\mu\right)\left(\mu_{i}-\mu\right)^{T}
$$

where

$\mu$ is the mean vector of the entire target class.

The primal optimization problem of the standard OSVM can be defined as [14], [23]:

$$
\begin{array}{r}
\min _{w, \xi, \rho} \frac{1}{2} w^{T} w+\frac{1}{v N} \sum_{i=1}^{N} \xi_{i}-\rho, \\
\text { s.t } w^{T} x_{i} \geq \rho-\xi_{i}, \xi_{i} \geq 0 \forall i=1, \ldots, N,
\end{array}
$$

where

$w$ is the weight vector of the separating hyperplane, $v \in[0,1]$ is a key parameter that acts as an upper bound for the fraction of outliers, and a lower bound for the fraction of Support Vectors (SVs) within the training dataset, $\xi_{i}$ is a slack variable, and $\rho$ is a threshold or bias term.

After adding the within and between subclasses matrix $M_{s u b}$ of (5) to the primal optimization problem of (8), the SDOSVM primal optimization problem can be defined as:

$$
\begin{gathered}
\min _{w, \xi, \rho} \frac{1}{2} w^{T} M_{s u b} w+\frac{1}{v N} \sum_{i=1}^{N} \xi_{i}-\rho, \\
\text { s.t } w^{T} M_{s u b} w>0, w^{T} x_{i} \geq \rho-\xi_{i}, \xi_{i} \geq 0 \forall i=1, \ldots, N .
\end{gathered}
$$

The Langrangian dual function can be used to solve the SDOSVM primal optimization problem. First, the SDOSVM primal optimization problem is reformulated to the Langrangian dual function as follows:

$L=\frac{1}{2} w^{T} M_{s u b} w+\frac{1}{v N} \sum_{i=1}^{N} \xi_{i}-\rho-\sum_{i=1}^{N} \alpha_{i}\left(w^{T} x_{i}+\xi_{i}-\rho\right)-\sum_{i=1}^{N} \beta_{i} \xi_{i}$,

where

$L$ is the Langrangian dual function, and $\alpha_{i}$ and $\beta_{i}$ are the Langrangian multipliers.

Second, the Langrangian dual function is solved by deriving it relatively to the variables $w, \xi$, and $\rho$ of the primal optimization problem, and the following is obtained:

$$
\begin{gathered}
w=M_{s u b}^{-1} \sum_{i=1}^{N} \alpha_{i} x_{i} . \\
\alpha_{i}=\frac{1}{v N}-\beta_{i} . \\
\sum_{i=1}^{N} \alpha_{i}=1 .
\end{gathered}
$$

Third, (11), (12), and (13) are substituted in (10), and the SDOSVM dual optimization problem is obtained:

$$
\begin{gathered}
\min _{\alpha} W(\alpha, \rho)=\frac{1}{2} \sum_{i=1}^{N} \sum_{j=1}^{N} \alpha_{i} \alpha_{j} K_{s u b}\left(x_{i}, x_{j}\right)+\rho\left(1-\sum_{i=1}^{N} \alpha_{i}\right), \\
\text { s.t } 0 \leq \alpha_{i} \leq \frac{1}{v N} \text {, and } \sum_{i=1}^{N} \alpha_{i}=1 \forall i=1, \ldots, N,
\end{gathered}
$$

where

$K_{s u b}=X^{T} M_{s u b}^{-1} X$ is the resulting kernel matrix, and $X=\left[x_{1}, \ldots, x_{N}\right]$ is the initial training matrix.

2) Nonlinear Expansion of SDOSVM: In order to distinguish between both the target and outlier classes using a nonlinear decision boundary, we need to project the classification from the input space $R^{I}$ to $R^{h}$ which is a higher dimension space such that $h>I$.

This can be achieved through the kernel trick where the inner product in (9) is substituted by the mapping kernel 
function $\phi$ such that each data point is mapped from the input space to the higher dimension space without altering any relations used in the input space.

Using the kernel trick, the SDOSVM primal optimization problem in (9) can be re-formulated as:

$$
\begin{gathered}
\min _{w, \xi, \rho} \frac{1}{2} w^{T} M_{s u b}^{\phi} w+\frac{1}{v N} \sum_{i=1}^{N} \xi_{i}-\rho, \\
\text { s.t } w^{T} M_{s u b}^{\phi} w>0, w^{T} \phi\left(x_{i}\right) \geq \rho-\xi_{i}, \xi_{i} \geq 0 \forall i=1, \ldots, N,
\end{gathered}
$$

where

$\phi\left(x_{i}\right)$ is the higher dimension class to which the target class is mapped using the mapping kernel function $\phi$.

$M_{W}^{s u b}$ and $M_{B}^{s u b}$ can be also re-formulated as:

$$
\begin{gathered}
M_{W}^{s u b^{\phi}}=\sum_{i=1}^{N_{s u b}} \sum_{j=1}^{N_{i}} p_{i}\left(\phi\left(x_{j}\right)-\mu_{i}^{\phi}\right)\left(\phi\left(x_{j}\right)-\mu_{i}^{\phi}\right)^{T}, \\
M_{B}^{s u b^{\phi}}=\sum_{i=1}^{N_{s u b}}\left(\mu_{i}^{\phi}-\mu^{\phi}\right)\left(\mu_{i}^{\phi}-\mu^{\phi}\right)^{T},
\end{gathered}
$$

where

$\mu_{i}^{\phi}$ is the mean vector of the $\mathrm{i}^{\text {th }}$ subclass in the higher dimension space,

and $\mu^{\phi}$ is the mean vector of the entire target class in the higher dimension space.

Since $M_{s u b}^{\phi}$ will be used for the nonlinear classification problem, and in order to compute it easily, the $M_{s u b}$ will be decomposed using the singular value decomposition as:

$$
M_{s u b}=V^{T} D V,
$$

where

$V$ is the orthonormal matrix that contains the eigenvectors of $M_{s u b}$,

and $D$ is the diagonal matrix that contains the computed eigenvalues of $M_{s u b}$.

After decomposing $M_{s u b}$, the resulting matrices can be used for the following transformation:

$$
Q=D^{\frac{-1}{2}} V
$$

Using the transformation in (19), each sample $x_{i}$ can be transformed to $x_{i}^{Q}=Q x_{i}$. As a result the following formula is generated:

$$
x_{i}^{Q^{T}} x_{j}^{Q}=x_{i}^{T} Q^{T} Q x_{j}=x_{i}^{T} M_{s u b}^{-1} x_{j} .
$$

Equation (20) is applied to the entire training dataset $X$, and the result is:

$X^{Q}=\left[x_{1}^{Q}, \ldots, x_{N}^{Q}\right]=\left[Q x_{1}, \ldots, Q x_{N}\right]=Q X$.

Using the above formula, the following is calculated:

$$
X^{Q^{T}} X^{Q}=X^{T} Q^{T} Q X=X^{T} M_{\text {sub }}^{-1} X .
$$

As a result, the Radial Basis Function (RBF) kernel function of the SDOSVM can be formulated as:

$$
K_{M_{s u b}-R B F}\left(x_{i}, x_{j}\right)=e^{\left(\frac{-g\left(x_{i}, x_{j}\right)}{2 \sigma^{2}}\right)},
$$

where

$g\left(x_{i}, x_{j}\right)=\left(x_{i}-x_{j}\right)^{T} M_{s u b}^{-1}\left(x_{i}-x_{j}\right)$.

After the SDOSVM classifier is constructed, the test phase is performed to classify $x$, an unseen data sample, through the following classification function:

$$
f(x)=\sum_{j=1}^{N_{s v}} \alpha_{j} K_{M_{s u b}-R B F}\left(x_{j}, x\right)+\rho,
$$

where

$N_{s v}$ is the number of SVs, $x_{j}$, acquired during the training step.

\section{Proposed Algorithm}

In this subsection, the proposed Deep SDOSVM intrusion detection algorithm is summarized in Algorithm 1.

$\overline{\text { Algorithm } 1 \text { The Deep SDOSVM Intrusion Detection Algo- }}$ rithm

Input: $X=\left\{x_{i}\right\}_{i=1}^{N}$

1) Feature Mapping: Transform values of categorical features to numeric ones.

2) Feature Selection: Select best features for the classification problem.

3) Clustering: Find the optimal number of clusters for the target class using the DynAE deep clustering model.

4) Feature Normalization: Normalization of features using (1).

5) Subclasses Matrix Calculation: Compute the subclasses matrix $\left(M_{s u b}\right)$ using (5), (6), and (7).

6) Train the Model: Train the SDOSVM model with RBF kernel matrix $\left(K_{M_{s u b}-R B F}\right)$ using (22) on the target class by solving the primal optimization problem in (15).

7) Testing the Model: Test the SDOSVM model using the classification function in (23) to distinguish between normal and attack data.

8) Assessing Performance: Compute the AUC, the number of SVs, and the training time.

Output: Predicted class label vector

\section{EXPERIMENTS}

In this section, a comparative evaluation of the proposed Deep SDOSVM intrusion detection system is presented. First, a description of the benchmark datasets used for experimentation is provided. Second, the performance metrics used for validating the proposed system are described briefly. Third, the experimental protocol is described in detail. Fourth, a brief description of relevant ML one-class classifiers that are compared with the proposed approach is provided. Finally, the obtained experimental results are discussed.

\section{A. Datasets Used}

In this subsection, we describe in detail the datasets used for the experimentations as illustrated in Table III 
TABLE II

REAL-WORLD IOT DATASETS USED FOR THE EXPERIMENTATION

\begin{tabular}{|l|l|l|l|l|}
\hline Reference & Dataset & $\begin{array}{l}\text { No. of } \\
\text { Target } \\
\text { Class } \\
\text { Instances } \\
\text { per } \\
\text { Subset }\end{array}$ & $\begin{array}{l}\text { No. of } \\
\text { Outlier } \\
\text { Class } \\
\text { Instances } \\
\text { per } \\
\text { Subset }\end{array}$ & $\begin{array}{l}\text { No. of } \\
\text { Best } \\
\text { Features } \\
\text { per } \\
\text { Subset }\end{array}$ \\
\hline 26 & $\begin{array}{l}\text { TON_IoT } \\
\text { (5 subsets) }\end{array}$ & 2500 & 2500 & 15 \\
\hline
\end{tabular}

The TON_IoT dataset includes four diverse data sources that were collected from Telemetry datasets of IoT and Industrial Internet of Things (IIoT) sensors, Operating systems datasets of Windows 7 and 10 as well as Ubuntu 14.04 and 18.04 TLS, and Network traffic datasets. The dataset was generated using a pragmatic and large-scale network designed in the Cyber Range and IoT Labs of UNSW Canberra. More details about the dataset can be found in [26]-[28], and the directories of the dataset can be found in [29].

For easy handling of the TON_IoT dataset, the author created Train_Test_datasets that consists of four datasets for evaluating the performance of cyber security applicationbased Artificial Intelligence (AI) and ML algorithms. For the experiments the Train_Test_Network_dataset is used that consists of 461,043 data samples divided to 300,000 normal and 161,043 attack data samples. The attacks included in the network dataset are Backdoor, Distributed Denial of Service (DDoS), Denial of Service (DoS), Injection, Man in The Middle (MiTM), Password Cracking, Ransomware, Scanning, and Cross-Site Scripting (XSS) [26].

In the experiments five subsets are used for each of the target and outlier classes. Each target class and outlier class subset was created using a random selection of 2500 normal and attack data samples, respectively, from the Train_Test_Network_dataset.

\section{B. Performance Metrics}

In this subsection, the performance metrics used for validating the proposed Deep SDOSVM intrusion detection system are described briefly. The response of each performance metrics to the proposed Deep SDOSVM intrusion detection system is described in detail in section IV-E

1) ROC Curve and AUC: The Receiver Operating Characteristic (ROC) curve is a visual evaluation metric for the performance of classifiers that measures the trade-off between FPR and TPR [7].

The ROC curve is a powerful metric due to its dependency on the FPR and TPR without taking into account the number of training or testing data samples [30].

The Area Under Curve (AUC) metric corresponds to the classifier's performance. It is generated by the ROC curve, and is summarized as a float number in the range $[0,1][2]$, [7].

2) P-value: Paired-sample t-test probability-values (pvalues) were computed per subset between the proposed Deep SDOSVM approach and each of the relevant ML one-class classifiers using the AUC values.
The p-values quantify the probability of whether the paired AUC values for the same subset are the same or not in order to elicit the improvement in classification performance by the proposed Deep SDOSVM approach over the relevant ML oneclass classifiers.

3) FPR and TPR: The FPR is the ratio of the number of outlier class samples misclassified as target class samples to the total number of outlier class samples in the testing dataset:

$$
F P R=\frac{F P}{F P+T N},
$$

where

$F P$ is the number of outlier class samples misclassified as target class samples,

and $T N$ is the number of outlier class samples classified correctly.

The TPR is the ratio of the number of target class samples classified correctly to the total number of target class samples in the testing dataset:

$$
T P R=\frac{T P}{T P+F N},
$$

where

$T P$ is the number of target class samples classified correctly, and $F N$ is the number of target class samples misclassified as outlier class samples.

4) Number of SVs and Training Time: Both the number of SVs and training time have been computed for the proposed Deep SDOSVM approach and relevant ML one-class classifiers to demonstrate that the proposed Deep SDOSVM approach outperforms these classifiers in terms of computational complexity.

\section{Experimental Protocol}

This subsection presents the experimental protocol used to assess the performance of the proposed Deep SDOSVM intrusion detection system in order to show the advantage of the Deep SDOSVM approach over the standard OSVM and other relevant ML one-class classifiers.

First, feature mapping was done to transform values in the categorical features of the experimental datasets to numeric ones. The feature mapping was performed by the author on the TON_IoT dataset using the unclass function of the $\mathrm{R}$ programming language [31].

Second, feature selection was performed to select the best features for the classification problem. For the TON_IoT dataset, the SelectKBest function was used from the sklearn.feature_selection library of the python programming language to select the best 15 features from the total 43 features of the dataset that gave best results for the proposed intrusion detection system.

Third, in order to find the optimal number of clusters for the experimental datasets, the DynAE deep clustering algorithm proposed by [9] was used.

For the TON_IoT dataset, the optimal number of clusters obtained for each subset is shown in Table 피 
TABLE III

OPTIMAL NUMBER OF CLUSTERS OBTAINED PER SUBSET FOR THE TON_IOT DATASET

\begin{tabular}{|l|l|}
\hline Subset & $\begin{array}{l}\text { Optimal Number of } \\
\text { Clusters }\end{array}$ \\
\hline 1 & 4 \\
\hline 2 & 4 \\
\hline 3 & 4 \\
\hline 4 & 4 \\
\hline 5 & 3 \\
\hline
\end{tabular}

Fourth, the experimental target class datasets and their subclasses were normalized in order to compute the subclasses matrix $M_{s u b}$. Fifth, a 10-fold cross-validation method was used for the experimental normalized target class datasets where each dataset was randomly divided into 10 subsets such that each time 9 subsets were used for training to optimize the proposed model, and one subset was added to an experimental normalized outlier class dataset for testing to validate the proposed model. For each fold the performance metrics, i.e. the AUC, the number of SVs generated by the model, and the training time were computed.

The 10-fold cross-validation method was used to show explicitly unbiased and reliable results for the proposed Deep SDOSVM approach, and select the optimal values for the SDOSVM hyperparameters $v$ of (15) and $\sigma$ of (22) over the dataset.

Finally, the average AUC, the average number of SVs, and the average training time of the 10 -fold cross-validation method were computed for the Deep SDOSVM to assess its performance against the standard OSVM, and the other relevant ML one-class classifiers.

For implementation, Google Colaboratory, MATLAB R2021a, R i386 3.6.3, and Spyder 5 were used. The SVM and Kernel Methods Matlab Toolbox [32] and the Data Description Toolbox [33] were used for implementing the one-class classifiers. The github code created by [34] was used for implementing the One-Class-Neural Network (OCNN) classifier. The github code created by [35] and modified by [36] was also used for implementing the DynAE deep clustering model.

\section{Classifiers}

In this subsection, we describe briefly the ML one-class classifiers used for the comparative analysis of the Deep SDOSVM approach.

The proposed Deep SDOSVM approach is compared against the following five state-of-the-art ML one-class classifiers:

1) Gaussian: The target class is modeled in this classifier by utilizing a single gaussian density. The mean and covariance matrix are estimated using maximum likelihood. In case of high dimensional data, a regularization term is added to the covariance matrix. There is no need to optimize parameters for this classifier [37].

2) Support Vector Data Description (SVDD): This classifier is inspired by the SVM. It fits a spherical boundary which has the minimal volume (or radius) around the target class, and this boundary is constructed using a set of SVs describing it. The implementation of this classifier used in experimentation has the RBF kernel hard-coded in order to ease implementation and evaluation [38].

3) OSVM: This classifier differs from the SVDD as the SVDD fits the hypersphere with minimal radius around the target class, where as the OSVM tries to find the hyperplane with maximum margin to separate the target class from the origin [37].

4) Mahalanobis One-class Support Vector Machine (MOSVM): This classifier is an extension of the OSVM. The Mahalanobis distance is used instead of the Euclidean distance as the distance-metric in order to utilize the data covariance as a matrix [39].

5) OC-NN: This classifier can be considered as a neural architecture utilizing the OSVM equivalent loss function. It utilizes a simple feed forward network with a single hidden layer having linear or sigmoid activation, and one output node. The linear activation is used in the experimentation [40].

6) SDOSVM: This classifier was built upon the OSVM as described in section III-C, and uses the K-means clustering algorithm to generate the subclasses as in [41].

\section{E. Results and Discussion}

This subsection presents the average results of the ten folds obtained from the conducted experiments on the five TON_IoT subsets for the proposed Deep SDOSVM and the relevant ML one-class classifiers which are summarised in Tables IV, V] VI, and Fig. 4 .

The improvement in performance by the proposed Deep SDOSVM approach over the relevant ML one-class classifiers is validated using the performance metrics described in subsection IV-B.

1) ROC Curve and AUC: As we can see from the obtained average AUC results in Table IV, both SDOSVM and Deep SDOSVM provide better AUC results for all five subsets than the other one-class SVM-based classifiers, namely, the MOSVM, the Standard OSVM, and the SVDD. This can be explained due to the fact that the SDOSVM approach considers subclasses in the target class, thus minimizing the data dispersion within and between subclasses, and improving the discriminative power and classification performance.

The SDOSVM is outperformed by the Deep SDOSVM for all subsets except for Subset-3, where both classifiers provide the same AUC result. This can be attributed to the usage of the K-means clustering algorithm, as in [41], by the SDOSVM to generate the subclasses based on Euclidean distance-based metrics, and these metrics are not adequate for the discovery of representative similarities between data points as they assume that the clusters are spherical, and therefore we cannot perform good clustering in spherical form. The DynAE clustering model used by the Deep SDOSVM is free from such an assumption on clusters, and therefore, generated better AUC results.

Embedded data representation and dimensionality reduction have been used alongside in order to project the input data 
TABLE IV

AVERAGE AUC PER SUBSET FOR THE TON_IOT DATASET (BEST METHOD IN BOLD)

\begin{tabular}{|l|l|l|l|l|l|l|l|l|l|l|l|}
\hline \multirow{2}{*}{ Subsets } & \multicolumn{3}{|c|}{$\mathbf{1}$} & \multicolumn{2}{|c|}{$\mathbf{2}$} & \multicolumn{2}{|c|}{$\mathbf{3}$} & \multicolumn{2}{|c|}{$\mathbf{4}$} & \multicolumn{2}{|c|}{$\mathbf{5}$} \\
\cline { 2 - 13 } & AUC & 1-p-value & AUC & 1-p-value & AUC & 1-p-value & AUC & 1-p-value & AUC & 1-p-value \\
\hline Gaussian & 75.96 & 100 & 79.13 & 100 & 79.86 & 100 & 76.14 & 100 & 84.36 & 100 \\
\hline SVDD & 94.86 & 100 & 93.90 & 100 & 93.59 & 100 & 94.20 & 100 & 94.12 & 100 \\
\hline OSVM & 92.86 & 100 & 92.43 & 100 & 92.14 & 100 & 92.98 & 100 & 92.95 & 100 \\
\hline MOSVM & 92.92 & 100 & 92.73 & 100 & 92.79 & 100 & 93.10 & 100 & 92.74 & 100 \\
\hline OC-NN & 75.95 & 100 & 76.19 & 100 & 78.11 & 100 & 77.15 & 100 & 76.08 & 100 \\
\hline SDOSVM & 97.14 & 99.94 & 99.49 & 99.96 & $\mathbf{9 9 . 9 2}$ & 0 & 99.25 & 98.56 & 96.91 & 56.76 \\
\hline Deep SDOSVM & $\mathbf{9 7 . 9 5}$ & - & $\mathbf{9 9 . 7 6}$ & - & $\mathbf{9 9 . 9 2}$ & - & $\mathbf{9 9 . 8 2}$ & - & $\mathbf{9 7 . 0 6}$ & - \\
\hline
\end{tabular}

into a lower dimensional feature space, where separation and similarity are better stressed with respect to the problem's context. For this reason, Autoencoders are considered to be a natural choice. In fact, Deep Embedded Clustering allows better analysis by training to represent the data as a nested hierarchy of concepts within layers of the Autoencoder, thereby allowing effective data dimension reduction and highly nonlinear decision separation between clusters at the Autoencoder bottleneck level.

Also, the Gaussian classifier is outperformed by the one-class SVM-based classifiers (MOSVM, OSVM, SVDD, SDOSVM, and Deep SDOSVM). This can be explained by the limitation of the Gaussian classifier as it assumes that the underlying distribution of the training data is Gaussian, and this case is not always true for real-world datasets. The oneclass SVM-based classifiers are free from such an assumption, and hence these classifiers generated better AUC results in all five subsets [37].

The Deep SDOSVM also outperforms the OC-NN in terms of AUC values for all five subsets. This can be explained due to the fact that the objective function for the OC-NN is nonconvex, and therefore, deducing the model parameters will not reach to a global optima [40].

Moreover, the average ROC curves for the Deep SDOSVM and the aforementioned one-class classifiers per subset are illustrated in Fig. 4. As observed, the ROC curves of the Deep SDOSVM are the best because it achieved the best tradeoff between the average FPR and TPR which resulted in the best AUC for all five subsets, and therefore, it has the best classification performance over the other one-class classifiers.

2) P-value: As observed from Table IV, all of the (1-pvalue) are approximately above $98.5 \%$ except for Subset-3 and Subset-5 where (1-p-value) for the SDOSVM is $0 \%$ and $56.76 \%$, respectively. Most of these values indicate a high probability that the paired AUC values of the Deep SDOSVM and each of the one-class classifiers are not the same for each of the five subsets, and therefore, the Deep SDOSVM provides statistically considerable improvement in performance over the other one-class classifiers.

3) FPR and TPR: In Table V, the average FPR and TPR for the Deep SDOSVM and the other one-class classifiers are presented per subset. As can be seen, the Deep SDOSVM provides the lowest FPR for all subsets, and the highest TPR for most subsets.

The SVDD provides better TPR than the Deep SDOSVM for Subset-5, but is considerably outperformed by the Deep
SDOSVM in terms of FPR. This can be explained due to the fact that the Deep SDOSVM considers subclasses in the target class in order to improve the discriminative power and classification performance. Also, the Deep SDOSVM uses the DynAE deep clustering model for subclasses generation to further enhance the classification performance of the intrusion detection system.

4) Number of SVs and Training Time: All the compared SVM-based classifiers including the proposed Deep SDOSVM scale with a complexity of $O\left(N^{2}\right)$, where $N$ represents the number of training samples. However, as seen from the obtained average SVs and training time results in Table VI both the Deep SDOSVM and SDOSVM have better average SVs and training time results compared to the other SVMbased classifiers for all subsets. This can be explained due to the fact that both approaches minimize the data dispersion, while projecting data, which reduces the number of SVs, and hence, results in lower computational complexity as indicated by the obtained average training time.

In particular, the MOSVM provides the highest training time, since it incorporates the inversion of both kernel matrix and covariance matrix, whereas for the Deep SDOSVM the inversion of the dispersion matrix is performed once in the input space (refer to the equations from (18) to (22)).

The Deep SDOSVM also outperforms the OC-NN classifier as seen in Table VI because of the high number of epochs, which is 100 for the experimentation, despite the use of backward propagation instead of the forward propagation.

Finally, as seen from Table VI the Gaussian classifier achieves better training time than the Deep SDOSVM and the other one-class classifiers, which does not exceed 0.06 seconds for all five subsets. This can be explained due to the fact that the Gaussian is a non-iterative algorithm unlike the Deep SDOSVM and the other one-class classifiers that use the gradient descent algorithm, and therefore, are iterative algorithms.

\section{CONClusion}

In this paper, we proposed a ML subclasses one-class anomaly-based network intrusion detection system for the IoHT, namely Deep SDOSVM, which is a novel variation of the standard OSVM that considers the subclasses in the IoHT normal data class in order to minimize data dispersion within and between subclasses thus improving the discriminative power and classification performance. 


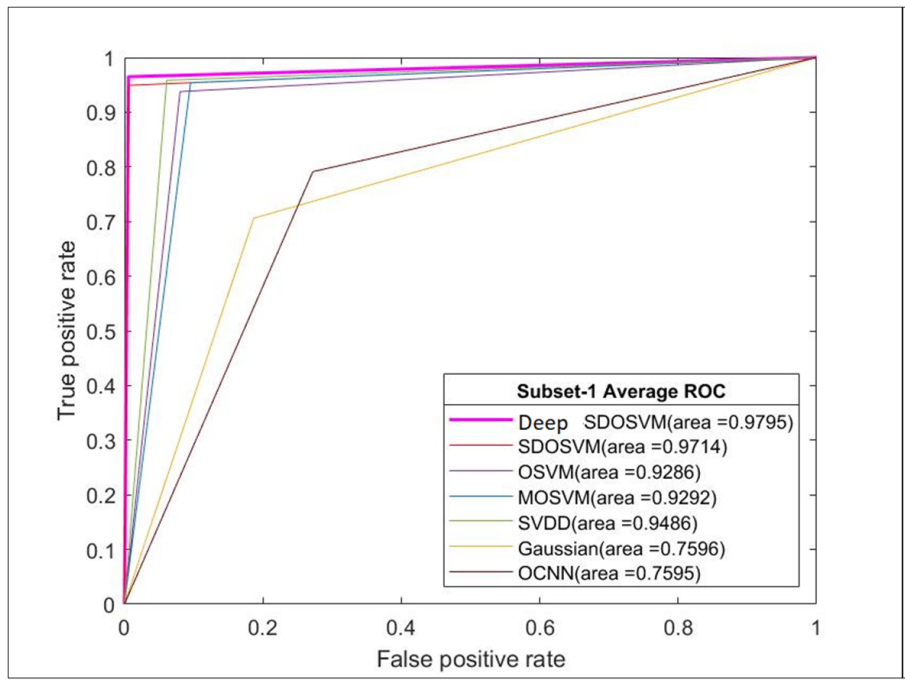

(a)

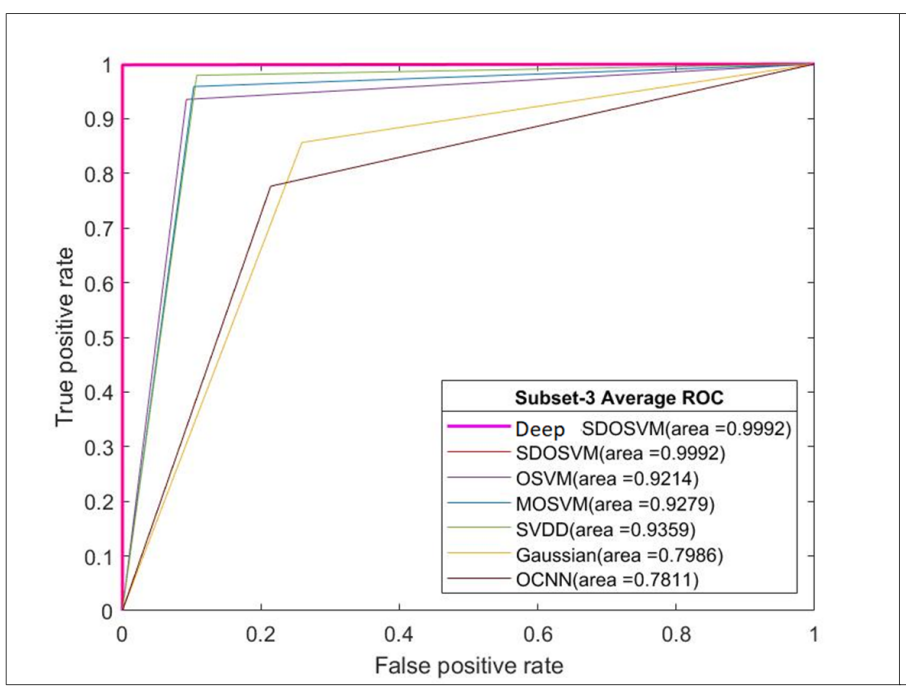

(c)

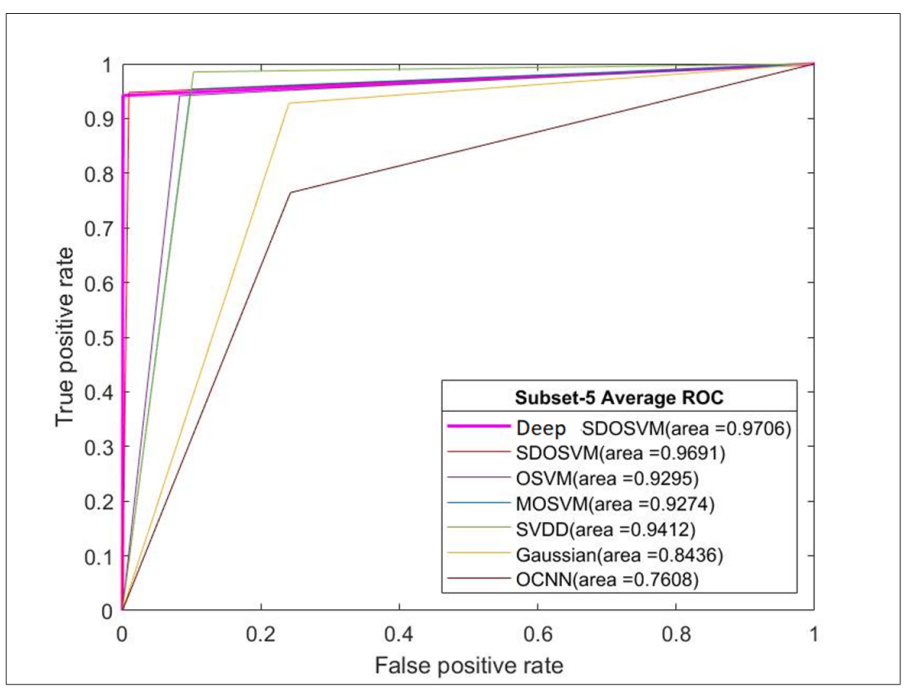

(e)

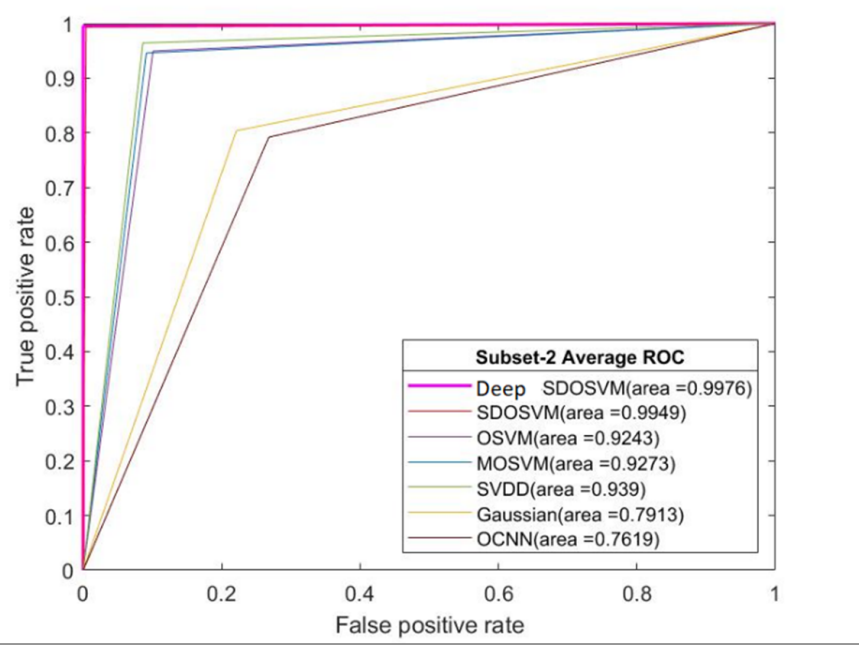

(b)

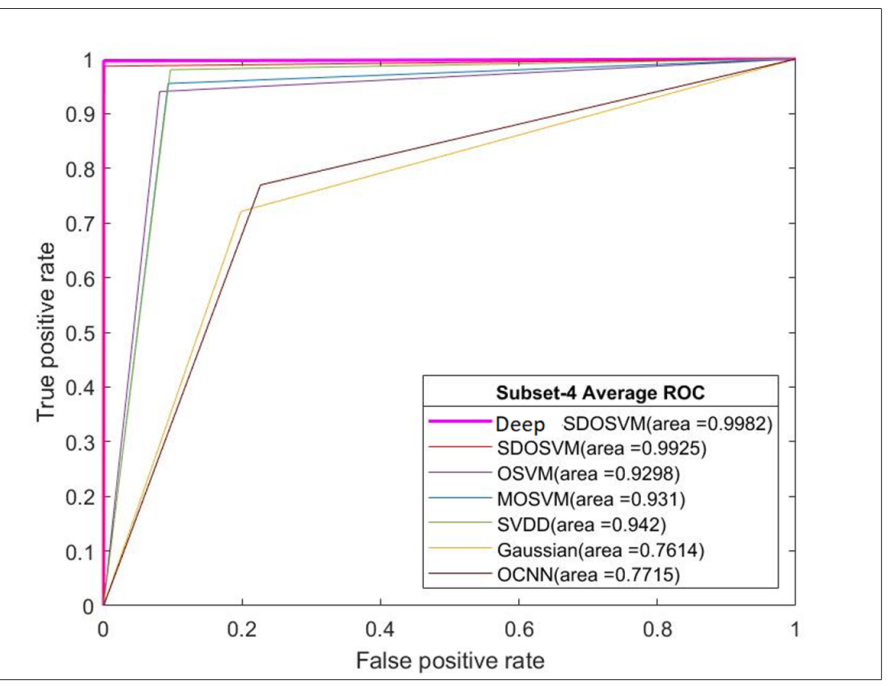

(d)

Fig. 4. Average ROC curves of one-class classifiers per subset for the TON_IoT dataset 
TABLE V

AVERAGE FPR AND TPR PER SUBSET FOR THE TON_IOT DATASET (BEST METHOD IN BOLD)

\begin{tabular}{|c|l|l|l|l|l|l|l|l|}
\hline \multicolumn{2}{|c|}{ Subsets } & Gaussian & SVDD & OSVM & MOSVM & OC-NN & SDOSVM & Deep SDOSVM \\
\hline \multirow{2}{*}{$\mathbf{1}$} & $\begin{array}{l}\text { Average } \\
\text { FPR }\end{array}$ & 18.67 & 6.11 & 8.04 & 9.56 & 27.22 & 0.64 & $\mathbf{0 . 5 8}$ \\
\cline { 2 - 8 } \\
$\begin{array}{l}\text { Average } \\
\text { TPR }\end{array}$ & 70.60 & 95.84 & 93.76 & 95.40 & 79.12 & 94.92 & $\mathbf{9 6 . 4 8}$ \\
\hline \multirow{2}{*}{$\mathbf{2}$} & $\begin{array}{l}\text { Average } \\
\text { FPR }\end{array}$ & 22.14 & 8.63 & 10.10 & 9.13 & 26.82 & 0.42 & $\mathbf{0 . 0 1}$ \\
\cline { 2 - 8 } \\
$\begin{array}{l}\text { Average } \\
\text { TPR }\end{array}$ & 80.40 & 96.44 & 94.96 & 94.60 & 79.20 & 99.40 & $\mathbf{9 9 . 5 2}$ \\
\hline \multirow{2}{*}{$\mathbf{3}$} & $\begin{array}{l}\text { Average } \\
\text { FPR }\end{array}$ & 25.93 & 10.74 & 9.24 & 10.30 & 21.42 & $\mathbf{0 . 0 0}$ & $\mathbf{0 . 0 0}$ \\
$\begin{array}{l}\text { Average } \\
\text { TPR }\end{array}$ & 85.64 & 97.92 & 93.52 & 95.88 & 77.64 & $\mathbf{9 9 . 8 4}$ & $\mathbf{9 9 . 8 4}$ \\
\hline \multirow{2}{*}{$\mathbf{4}$} & $\begin{array}{l}\text { Average } \\
\text { FPR }\end{array}$ & 19.84 & 9.64 & 8.09 & 9.32 & 22.66 & 0.17 & $\mathbf{0 . 0 0}$ \\
\cline { 2 - 8 } \\
$\begin{array}{l}\text { Average } \\
\text { TPR }\end{array}$ & 72.12 & 98.04 & 94.04 & 95.52 & 76.96 & 98.68 & $\mathbf{9 9 . 6 4}$ \\
\hline \multirow{2}{*}{$\mathbf{5}$} & $\begin{array}{l}\text { Average } \\
\text { FPR }\end{array}$ & 24.08 & 10.28 & 8.26 & 9.88 & 24.27 & 0.94 & $\mathbf{0 . 0 8}$ \\
\cline { 2 - 8 } & $\begin{array}{l}\text { Average } \\
\text { TPR }\end{array}$ & 92.80 & $\mathbf{9 8 . 5 2}$ & 94.16 & 95.36 & 76.44 & 94.76 & 94.20 \\
\hline
\end{tabular}

TABLE VI

AVERAGE NUMBER OF SUPPORT VECTORS AND TRAINING TIME PER SUBSET FOR THE TON_IOT DATASET (BEST RESULT IN BOLD)

\begin{tabular}{|c|c|c|c|c|c|c|c|c|}
\hline \multicolumn{2}{|c|}{ Subsets } & Gaussian & SVDD & OSVM & MOSVM & OC-NN & SDOSVM & Deep SDOSVM \\
\hline \multirow[t]{2}{*}{1} & $\begin{array}{l}\text { Average } \\
\text { SVs }\end{array}$ & - & 2250 & 171 & 938 & - & 121 & 91 \\
\hline & $\begin{array}{l}\text { Average } \\
\text { Training } \\
\text { Time (s) }\end{array}$ & 0.02 & 18.88 & 1.39 & 23.06 & 3.45 & 0.99 & 0.76 \\
\hline \multirow[t]{2}{*}{2} & $\begin{array}{l}\text { Average } \\
\text { SVs }\end{array}$ & - & 2250 & 124 & 974 & - & 19 & 13 \\
\hline & $\begin{array}{l}\text { Average } \\
\text { Training } \\
\text { Time (s) }\end{array}$ & 0.01 & 18.09 & 1.00 & 21.26 & 3.89 & 0.36 & 0.33 \\
\hline \multirow[t]{2}{*}{3} & $\begin{array}{l}\text { Average } \\
\text { SVs }\end{array}$ & - & 2250 & 149 & 969 & - & 5 & 5 \\
\hline & $\begin{array}{l}\text { Average } \\
\text { Training } \\
\text { Time (s) }\end{array}$ & 0.06 & 19.36 & 1.42 & 22.04 & 3.28 & 0.37 & 0.25 \\
\hline \multirow[t]{2}{*}{4} & $\begin{array}{l}\text { Average } \\
\text { SVs }\end{array}$ & - & 2250 & 151 & 901 & - & 38 & 12 \\
\hline & $\begin{array}{l}\text { Average } \\
\text { Training } \\
\text { Time (s) }\end{array}$ & 0.01 & 22.30 & 1.28 & 27.23 & 3.68 & 0.51 & 0.36 \\
\hline \multirow[t]{2}{*}{5} & $\begin{array}{l}\text { Average } \\
\text { SVs }\end{array}$ & - & 2250 & 137 & 968 & - & 116 & 132 \\
\hline & $\begin{array}{l}\text { Average } \\
\text { Training } \\
\text { Time (s) }\end{array}$ & 0.01 & 19.22 & 1.07 & 26.82 & 3.66 & 0.85 & 0.93 \\
\hline
\end{tabular}

The DynAE deep clustering model was used for subclasses generation in the proposed Deep SDOSVM approach to overcome the drawbacks of classical clustering algorithms such as $\mathrm{K}$-means, and further enhance the classification performance of the intrusion detection system.

Experimentation results based on the real-world IoT dataset, TON_IoT, showed that the proposed Deep SDOSVM approach outperformed the standard OSVM and other relevant ML oneclass classifiers in terms of AUC, where the AUC scores achieved by the proposed approach are above $97 \%$.

Also, the proposed approach outperformed the one-class SVM-based classifiers which are the MOSVM, OSVM, SVDD, and SDOSVM in terms of training time, which did not exceed one second, due to its reliance on a low number of SVs thus resulting in lower computational complexity.

Finally, the proposed approach achieved the best results than the one-class classifiers for the FPR, which did not exceed $0.6 \%$, and the TPR in most evaluation subsets of the TON_IoT dataset, which was above $96.4 \%$.

Based on the obtained results, the proposed Deep SDOSVM approach can be considered suitable for intrusion detection in the IoHT environments as these environments are heterogeneous, i.e. the definition of normal behavior can differ from one system to another, and resource-constraint.

For future work, the proposed Deep SDOSVM intrusion detection system will be modified to an incremental intrusion detection system for the IoHT in order to overcome limitations of being a batch learning model. The incremental version 
should be able to handle sequential obtained data, and largescale datasets without any degradation in performance, and manage the learning process at the beginning with few available data samples.

\section{ACKNOWLEDGMENT}

The authors would like to thank Khaoula Tbarki from the National Engineers School of Tunis (ENIT), University of Tunis El Manar, and Elham Eskandarnia from the British University of Bahrain for their assistance in developing this research work.

\section{REFERENCES}

[1] P. Ghosal, D. Das, and I. Das, "Extensive survey on cloud-based iot-healthcare and security using machine learning," in 2018 Fourth International Conference on Research in Computational Intelligence and Communication Networks (ICRCICN), 2018, pp. 1-5.

[2] P. Kumar, G. P. Gupta, and R. Tripathi, "An ensemble learning and fog-cloud architecture-driven cyber-attack detection framework for iomt networks," Computer Communications, vol. 166, pp. 110-124, 2021.

[3] G. Thamilarasu, A. Odesile, and A. Hoang, "An intrusion detection system for internet of medical things," IEEE Access, vol. 8, pp. 181560 181576,2020

[4] S. P. R.M., P. K. R. Maddikunta, P. M., S. Koppu, T. R. Gadekallu, C. L. Chowdhary, and M. Alazab, "An effective feature engineering for dnn using hybrid pca-gwo for intrusion detection in iomt architecture," Computer Communications, vol. 160, pp. 139-149, 2020.

[5] J.-P. A. Yaacoub, M. Noura, H. N. Noura, O. Salman, E. Yaacoub, R. Couturier, and A. Chehab, "Securing internet of medical things systems: Limitations, issues and recommendations," Future Generation Computer Systems, vol. 105, pp. 581-606, 2020.

[6] N. Chaabouni, M. Mosbah, A. Zemmari, C. Sauvignac, and P. Faruki, "Network intrusion detection for iot security based on learning techniques," IEEE Communications Surveys Tutorials, vol. 21, no. 3, pp. 2671-2701, 2019.

[7] A. A. Hady, A. Ghubaish, T. Salman, D. Unal, and R. Jain, "Intrusion detection system for healthcare systems using medical and network data: A comparison study," IEEE Access, vol. 8, pp. 106 576-106 584, 2020

[8] M. A. Al-Garadi, A. Mohamed, A. K. Al-Ali, X. Du, I. Ali, and M. Guizani, "A survey of machine and deep learning methods for internet of things (iot) security," IEEE Communications Surveys Tutorials, vol. 22, no. 3, pp. 1646-1685, 2020.

[9] N. Mrabah, N. M. Khan, R. Ksantini, and Z. Lachiri, "Deep clustering with a dynamic autoencoder: From reconstruction towards centroids construction," Neural Networks, vol. 130, pp. 206-228, 2020.

[10] M. AL-Hawawreh, N. Moustafa, and E. Sitnikova, "Identification of malicious activities in industrial internet of things based on deep learning models," Journal of Information Security and Applications, vol. 41, pp. $1-11,2018$.

[11] I. Alrashdi, A. Alqazzaz, R. Alharthi, E. Aloufi, M. A. Zohdy, and H. Ming, "Fbad: Fog-based attack detection for iot healthcare in smart cities," in 2019 IEEE 10th Annual Ubiquitous Computing, Electronics Mobile Communication Conference (UEMCON), 2019, pp. 0515-0522.

[12] M. Bagaa, T. Taleb, J. B. Bernabe, and A. Skarmeta, "A machine learning security framework for iot systems," IEEE Access, vol. 8, pp. $114066-$ $114077,2020$.

[13] F. A. Bakhtiar, E. S. Pramukantoro, and H. Nihri, "A lightweight ids based on $\mathrm{j} 48$ algorithm for detecting dos attacks on iot middleware," in 2019 IEEE 1st Global Conference on Life Sciences and Technologies (LifeTech), 2019, pp. 41-42.

[14] V. H. Bezerra, V. G. T. da Costa, S. Barbon Junior, R. S. Miani, and B. B. Zarpelão, "Iotds: A one-class classification approach to detect botnets in internet of things devices," Sensors, vol. 19, no. 14, 2019.

[15] E. Ciklabakkal, A. Donmez, M. Erdemir, E. Suren, M. K. Yilmaz, and P. Angin, "Artemis: An intrusion detection system for mqtt attacks in internet of things," in 2019 38th Symposium on Reliable Distributed Systems (SRDS), 2019, pp. 369-3692.

[16] G. Efstathopoulos, P. R. Grammatikis, P. Sarigiannidis, V. Argyriou, A. Sarigiannidis, K. Stamatakis, M. K. Angelopoulos, and S. K. Athanasopoulos, "Operational data based intrusion detection system for smart grid," in 2019 IEEE 24th International Workshop on Computer Aided Modeling and Design of Communication Links and Networks (CAMAD), 2019, pp. 1-6.
[17] M. Eskandari, Z. H. Janjua, M. Vecchio, and F. Antonelli, "Passban ids: An intelligent anomaly-based intrusion detection system for iot edge devices," IEEE Internet of Things Journal, vol. 7, no. 8, pp. 6882-6897, 2020.

[18] P. Kamble and A. Gawade, "Digitalization of healthcare with iot and cryptographic encryption against dos attacks," in 2019 International Conference on contemporary Computing and Informatics (IC3I), 2019, pp. 69-73.

[19] A. Khraisat, I. Gondal, P. Vamplew, J. Kamruzzaman, and A. Alazab, "A novel ensemble of hybrid intrusion detection system for detecting internet of things attacks," Electronics, vol. 8, no. 11, 2019.

[20] S. Liaqat, A. Akhunzada, F. S. Shaikh, A. Giannetsos, and M. A. Jan, "Sdn orchestration to combat evolving cyber threats in internet of medical things (iomt)," Computer Communications, vol. 160, pp. 697705, 2020.

[21] A. M. Said, A. Yahyaoui, F. Yaakoubi, and T. Abdellatif, "Machine learning based rank attack detection for smart hospital infrastructure," in The Impact of Digital Technologies on Public Health in Developed and Developing Countries, M. Jmaiel, M. Mokhtari, B. Abdulrazak, H. Aloulou, and S. Kallel, Eds., 2020, pp. 28-40.

[22] M. Sheikhan and H. Bostani, "A hybrid intrusion detection architecture for internet of things," in 2016 8th International Symposium on Telecommunications (IST), 2016, pp. 601-606.

[23] A. A. Shorman, H. Faris, and I. Aljarah, "Unsupervised intelligent system based on one class support vector machine and grey wolf optimization for iot botnet detection," Journal of Ambient Intelligence and Humanized Computing, vol. 11, p. 2809-2825, 2020.

[24] A. Yahyaoui, T. Abdellatif, and R. Attia, "Hierarchical anomaly based intrusion detection and localization in iot," in 2019 15th International Wireless Communications Mobile Computing Conference (IWCMC), 2019, pp. 108-113.

[25] P. Singh, S. Krishnamoorthy, A. Nayyar, A. K. Luhach, and A. Kaur, "Soft-computing-based false alarm reduction for hierarchical data of intrusion detection system," International Journal of Distributed Sensor Networks, vol. 15, no. 10, p. 1550147719883132, 2019.

[26] N. Moustafa, "A new distributed architecture for evaluating ai-based security systems at the edge: Network ton_iot datasets," Sustainable Cities and Society, vol. 72, p. 102994, 2021. [Online]. Available: https://www.sciencedirect.com/science/article/pii/S2210670721002808

[27] T. M. Booij, I. Chiscop, E. Meeuwissen, N. Moustafa, and F. T. H. den Hartog, "Ton_iot: The role of heterogeneity and the need for standardization of features and attack types in iot network intrusion datasets," IEEE Internet of Things Journal, pp. 1-1, 2021.

[28] N. Moustafa, "New generations of internet of things datasets for cybersecurity applications based machine learning: Ton_iot datasets," in Proceedings of the eResearch Australasia Conference, 2019.

[29] N. Mostafa, "The ton_iot datasets," 2019, last accessed 21 June 2021. [Online]. Available: https://cloudstor.aarnet.edu.au/plus/ s/ds5zW91vdgjEj9i

[30] A. Besrour and R. Ksantini, "Incremental subclass support vector machine," International Journal on Artificial Intelligence Tools, vol. 28, no. 07 , p. $1950020,2019$.

[31] N. Moustafa, "Ton_iot network dataset," 2020, last accessed 21 June 2021. [Online]. Available: https://github.com/Nour-Moustafa/ TON_IoTNetworkdataset

[32] S. Canu, Y. Grandvalet, V. Guigue, and A. Rakotomamonjy, "Svm and kernel methods matlab toolbox," Perception Systems et Information, INSA de Rouen, Rouen, France, 2005, last accessed 21 June 2021. [Online]. Available: http://asi.insa-rouen.fr/enseignants/ arakoto/ toolbox/

[33] D. Tax, "Data description toolbox," 2013, last accessed 21 June 2021. [Online]. Available: http://prtools.tudelft.nl/Guide/37Pages/ software.html

[34] R. Chalapathy, "oc-nn," 2018, last accessed 1 September 2021. [Online]. Available: https://github.com/wzmsltw/oc-nn

[35] N. Mrabah, "Dynae," 2021, last accessed 25 January 2022. [Online]. Available: https://github.com/nairouz/DynAE

[36] E. Eskandarnia, H. M. Al-Ammal, and R. Ksantini, "An embedded deep-clustering-based load profiling framework," Sustainable Cities and Society, vol. 78, p. 103618, 2022.

[37] N. Mefraz Khan, R. Ksantini, I. Shafiq Ahmad, and L. Guan, "Covariance-guided one-class support vector machine," Pattern Recognition, vol. 47, no. 6, pp. 2165-2177, 2014.

[38] D. M. Tax and R. P. Duin, "Support vector domain description," Pattern Recognition Letters, vol. 20, no. 11, pp. 1191-1199, 1999. 
[39] I. Tsang, J. Kwok, and S. Li, "Learning the kernel in mahalanobis oneclass support vector machines," in The 2006 IEEE International Joint Conference on Neural Network Proceedings, 2006, pp. 1169-1175.

[40] R. Chalapathy, A. K. Menon, and S. Chawla, "Anomaly detection using one-class neural networks," CoRR, vol. abs/1802.06360, 2018. [Online]. Available: http://arxiv.org/abs/1802.06360

[41] K. Tbarki, R. Ksantini, S. B. Said, and Z. Lachiri, "A novel landmine detection system based on within and between subclasses dispersion information," International Journal of Remote Sensing, vol. 42, no. 19 , pp. 7405-7427, 2021. 